\title{
Glucosinolates and their hydrolysis products as potential nutraceuticals to combat cytokine storm in SARS-COV-2
}

\author{
Saba Rahimi Bahoosh ${ }^{1} \cdot$ Yalda Shokoohinia ${ }^{2} \cdot$ Mahdieh Eftekhari ${ }^{3,4} \mathbb{D}$
}

Received: 17 October 2021 / Accepted: 23 January 2022 / Published online: 2 February 2022

(c) Springer Nature Switzerland AG 2022

\begin{abstract}
Introduction The high mortality rate in severe cases of COVID-19 is mainly due to the strong upregulation of cytokines, called a cytokine storm. Hyperinflammation and multiple organ failure comprise the main clinical features of a cytokine storm. Nrf2 is a transcription factor which regulates the expression of genes involved in immune and inflammatory processes. Furthermore, Nrf2, as a master regulator, controls the activity of NF- $\mathrm{kB}$ which binds to the promoter of many pro-inflammatory genes inducible of various inflammatory factors. Inhibition of Nrf2 response was recently demonstrated in biopsies from patients with COVID-19, and Nrf2 agonists inhibited SARS-CoV-2 replication across cell lines in vitro. Glucosinolates and their hydrolysis products have excellent anti-inflammatory and antioxidant effects via the Nrf2 activation pathway, reduction in the NF-KB activation, and subsequent reduced cytokines levels.
\end{abstract}

Conclusion Accordingly, these compounds can be helpful in combating the cytokine storm associated with COVID-19.

Keywords SARS-COV-2 $\cdot$ COVID-19 $\cdot$ Glucosinolates $\cdot$ Nrf2 $\cdot$ NF-kappa B $\cdot$ Cytokine storm

\section{Introduction}

Severe acute respiratory syndrome coronavirus 2 (SARS$\mathrm{CoV}-2$ ) is the causative agent of a viral infectious disease called COVID-19, which was first identified in late 2019 in Wuhan, China and has now spread to all over the world [1]. The upper respiratory tract is the main location of primary infection after which the virus may infect other organs such as the kidneys, liver, and heart [2]. Evidence

Mahdieh Eftekhari

Mahdieh.eftekhari@gmail.com;

mahdieh.eftekhari@kums.ac.ir

1 Student Research Committee, Kermanshah University of Medical Sciences, Kermanshah, Iran

2 Ric Scalzo Institute for Botanical Research, Southwest College of Naturopathic Medicine, Tempe, AZ 85282, USA

3 Pharmaceutical Sciences Research Center, Kermanshah University of Medical Sciences, 6715847141 Kermanshah, Iran

4 Department of Pharmacognosy and Pharmaceutical Biotechnology, Faculty of Pharmacy, Kermanshah University of Medical Sciences, P.O.BOX.6714415153, Kermanshah, Iran suggests that overproduction of reactive oxygen species (ROS) and depletion of antioxidant power are crucial for the progression of SARS-CoV-2 infection [3]. Research has also indicated that the main cause of pathogenicity and mortality in critically ill patients with COVID-19 is the elevated inflammatory response triggered by the virus [1]. At the onset of the disease, the immune system fights the virus and controls inflammation by releasing a moderate amount of cytokines, but over time, the immune system may damage host cells by over-releasing cytokines. Thus, pathological complications arise due to an imbalance between the 'good' and the 'bad' immune systems [4]. Excessive production of cytokines by the overactive immune system leads to a condition called a cytokine storm; this is the main cause of health deterioration in patients with COVID-19 presenting with main clinical features of hyperinflammation, hyperferritinemia, and multiple organ failure [1]. During a cytokine storm, we see an excessive increase in the activity of immune system cells, especially macrophages and monocytes, followed by a significant increase in the release of cytokines such as interleukin (IL)-6, IL-17, IL-7, IL-1 $\beta$, IL-9, IL-2, IL-10 and tumor necrosis factor (TNF)- $\alpha[1,5,6]$ 


\section{Conventional therapy for COVID-19}

Since the beginning of the COVID-19 epidemic, many antiviral drugs have been tested and developed against SARS-CoV-2, but no specific drug has yet been found. In addition, some drugs have been used to stop COVID19-associated cytokine storm [3, 5]. Monoclonal antibodies have been shown to be useful in managing the cytokine storm induced by COVID-19, for example, the drug tocilizumab, an IL-6 inhibitor. Tocilizumab is FDAapproved for the treatment of autoimmune diseases such as rheumatoid arthritis. Among monoclonal antibodies, clazakizumab is another candidate for COVID-19-related cytokine management, however this drug has not been approved by the FDA for use in treatment [6]. Corticosteroids including dexamethasone inhibit the cytokine levels and have anti-inflammatory effects; therefore, they may be helpful in combating the cytokine storm in COVID-19 patients [5].

\section{Nrf2 pathway \& COVID-19}

Recently, in biopsies of COVID-19 patients, the suppression of the nuclear factor-erythroid 2-related factor-2 (Nrf2) response has been demonstrated while the Nrf2 agonists inhibited SARS-CoV-2 replication across cell lines in vitro [7]. There is also evidence of the antiviral effect of Nrf2 on respiratory infections caused by SARSCoV-2 [8]. Findings from several studies suggest that activation of Nrf2 may modulate the immune system. Nrf2 exerts its effects by regulating the transcription of various genes [9]. Nrf2 plays a central role in protecting cells against oxidative damage [10]. Nrf2 normally binds to its inhibitor in the cytoplasm, but under conditions of oxidative stress and in the presence of ROS, it separates from its inhibitor and migrates into the nucleus to control the expression of genes involved in the inflammatory process. Thus, Nrf2 increases the breakdown of pro-inflammatory factors and levels of anti-inflammatory agents, and thereby reduces ROS production, leading to protection against oxidative stress. Other benefits include reducing the severity of inflammation and increasing the production of antioxidant enzymes [11]. Concurrently, Nrf2 controls the activity of nuclear factor kappa-light-chain-enhancer of activated B cells $(\mathrm{NF}-\kappa \mathrm{B})[10]$. NF- $\kappa \mathrm{B}$ is a major transcription factor that binds to promoters of many pro-inflammatory inducible genes including TNF- $\alpha$, IL-6, IL- 8 , IL- $1 \beta$, nitric oxide synthase (NOS), and cyclooxygenase-2 (COX2) $[10,12-14]$. Based on clinical trials, Nrf2-activator molecules could be useful in combating the COVID-19 pandemic through prevention, diagnosis and treatment [15].

\section{Glucosinolates (GSs) and glucosinolate hydrolysis products (GSHPs)}

Mortality rate caused by COVID-19 has been low in Eastern Asia. One of the factors that contribute to the low mortality rate is the higher consumption of cruciferous vegetables, such as cabbage or radish, which are rich in GSs [16]. Brassica or cruciferous vegetables are rich in glucosinolates. These plants contain an enzyme called myrosinase, which hydrolyzes glucosinolates during tissue damage. Isothiocyanates (ITC) is one of the products of this reaction. ITC are responsible for the strong flavor of cruciferous vegetables such as broccoli sprouts or radishes [17]. Additionally, ITCs are well-known for their cancer-preventive effects and immune modulatory potential [18]. The various types of ITCs produced by the hydrolysis of GSs are depicted in Table 1 [17, 19]. GSs and GSHPs have shown great anti-inflammatory and antioxidant effects via activation Nrf2 pathway and reduction in the NF-kB activation and subsequent reduction in TNF- $\alpha$, IL-6 levels $[10,19]$.

\section{Sulforaphane (SFN) and Erucin (ER)}

SFN is the most studied ITC. A number of clinical studies have shown the beneficial effects of SFN in the prevention and treatment of cancers including lung cancer, liver cancer, leukemia, and breast cancer as well as others, It is also useful for the treatment of autoimmune diseases such as rheumatoid arthritis and colitis via suppressing the immune system [20]. In acute inflammatory lung injury, supplementation with SFN and oxygen therapy can be helpful [21]. Moreover, the beneficial effects of SFN in the prevention of asthma and chronic obstructive pulmonary disease (COPD) have been demonstrated [22]. For viral infections such as hepatitis $\mathrm{C}$, Acquired immune deficiency syndrome (AIDS) and the influenza, SFN can also effectively treat diseases through the Nrf2 pathway by inhibiting pro-inflammatory cytokines and activating antioxidant enzymes in monocytes and macrophages [20]. Furthermore, there is evidence that SFN reduces the transition of $N F-\kappa B$ to the nucleus and directly inactivates NF- $\mathrm{BB}$ subunits that can suppress the production of major pro-inflammatory mediators, 
Table 1 The structure of glucosinolates and their hydrolysis products

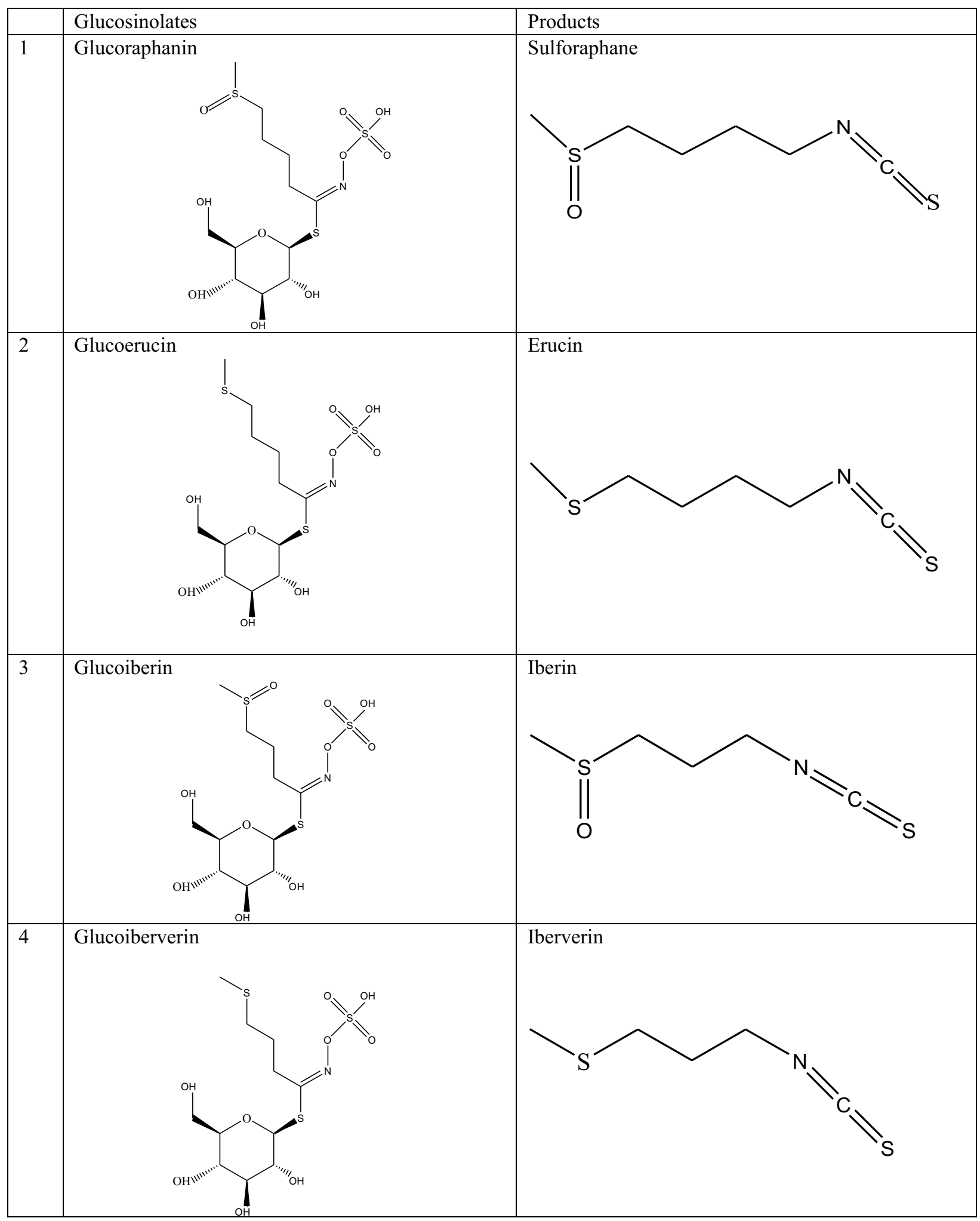


Table 1 (continued)

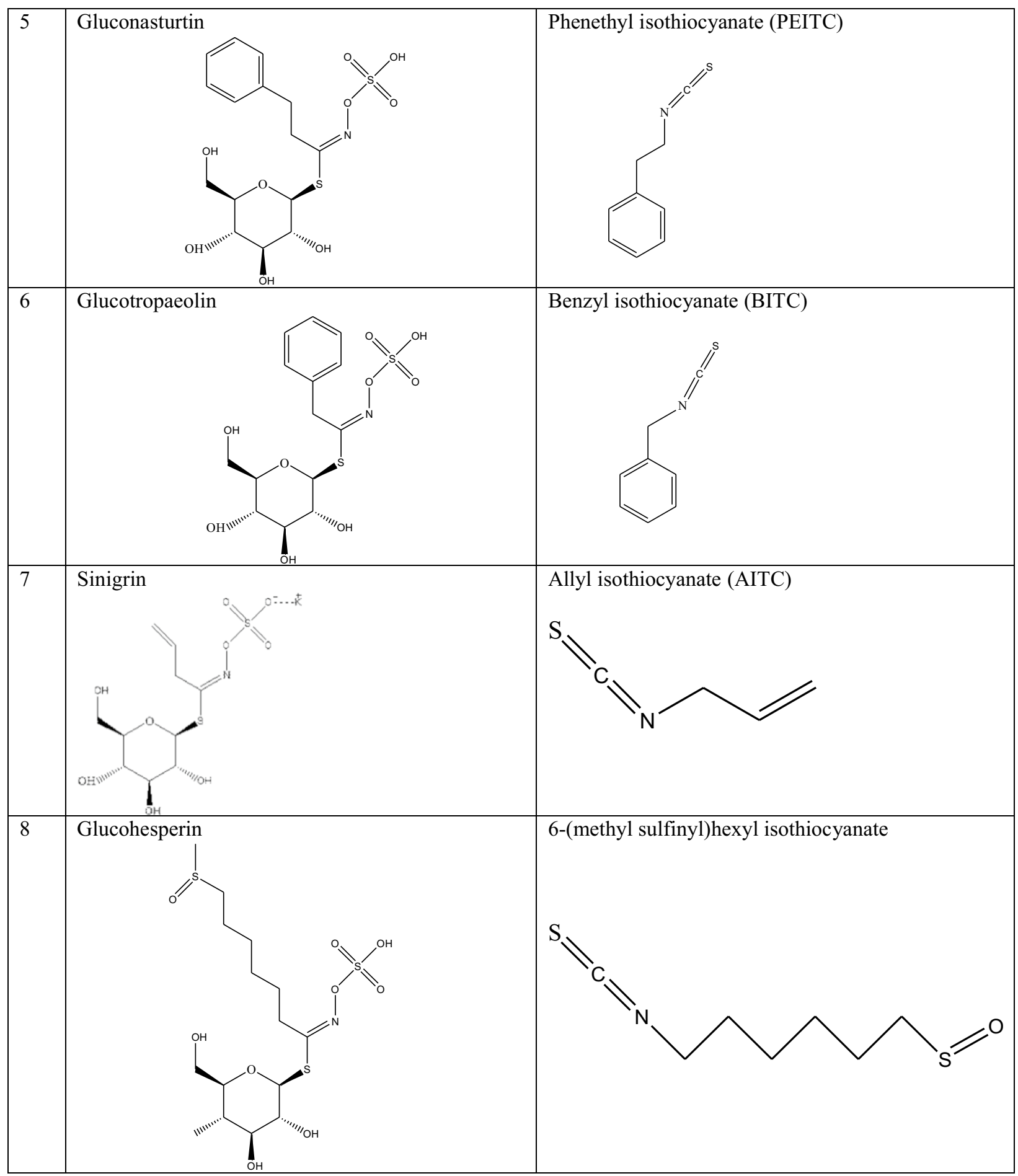

cytokines, and oxidative markers [10,23]. Another study has revealed that SFN, as a Nrf2 activator, can inhibit the expression of IL-6 and IL-8, which are induced by exposure to spike protein of SARS-CoV-2 in bronchial epithelial IB3-1 cells [24]. Other ITC, ER derived from glucoerucin is generally found in broccoli sprouts and arugula and can also be formed by reduction of SFN in vivo. ER, like SFN, inhibits 
Table 1 (continued)

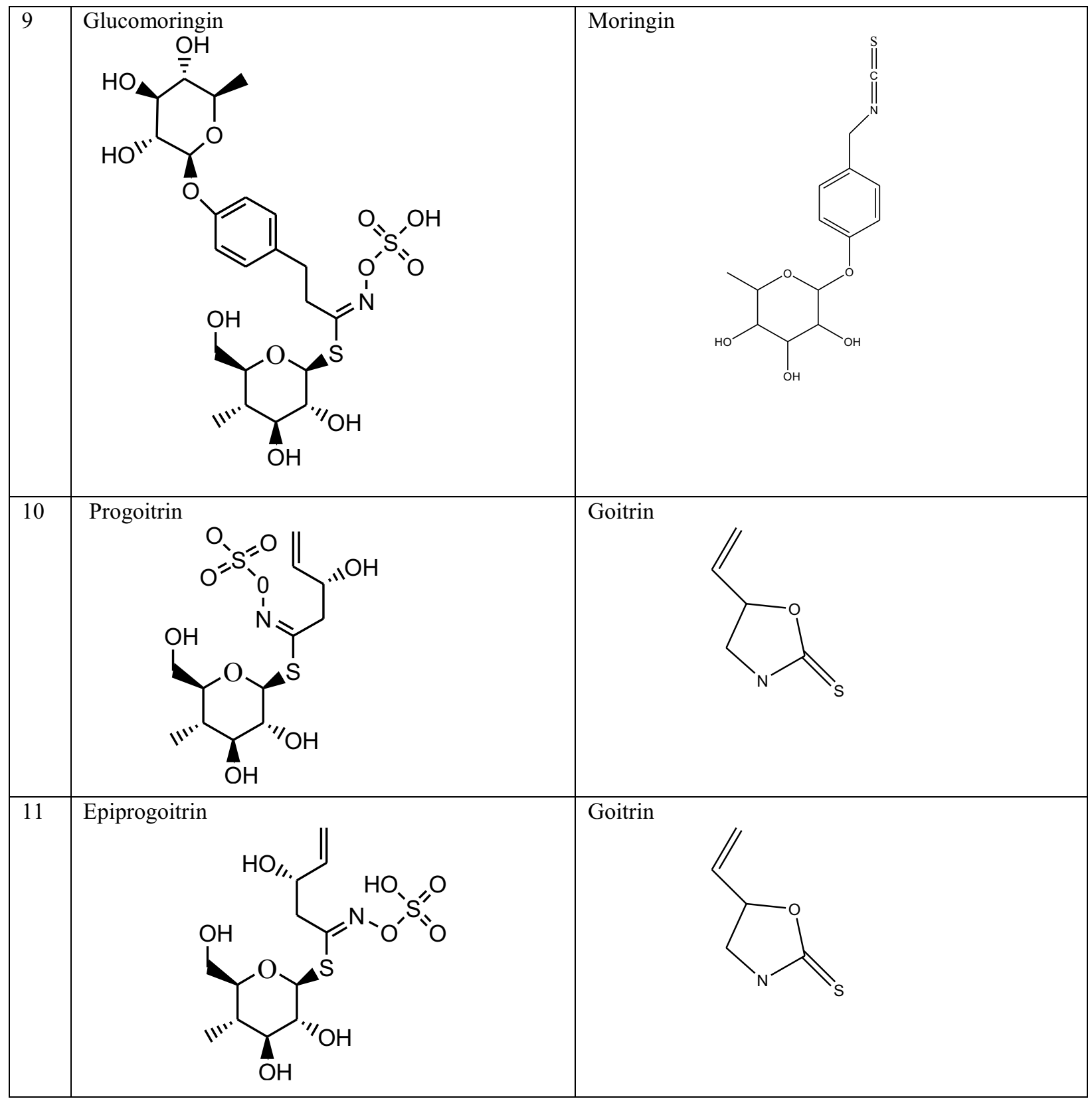

the NF- $\kappa \mathrm{B}$ signaling pathway as an anti-inflammatory agent and decreases the transcription of pro-inflammatory molecules (TNF- $\alpha$, IL-1 $\beta$ and IL-12) [12, 25].

\section{Iberin and Iberverin}

Glucoiberin and glucoiberverin are other GSs found in the plants of family Cruciferae, such as kale, brussels sprouts, broccoli seeds, and savoy cabbage. ITCs generated by myrosinase-catalyzed hydrolysis of these GSs are iberin and iberverin. They are homologues of sulforaphane and have similar potency to SFN in inducing Nrf2-dependent gene expression [26, 27].

\section{Phenethyl isothiocyanate (PEITC)}

PEITC results from myrosinase-catalyzed hydrolysis of gluconasturtin and is found predominantly in cruciferous plants such as horseradish [25]. PEITC like SFN has anticancer properties through various mechanisms [14, 
25]. PEITC was reported to influence the expression and nuclear translocation of Nrf2. Furthermore, PEITC inhibits NF- $\kappa B$ transcriptional activity that results in reducing inducible NOS (iNOS) and COX-2 protein expression levels leading to reduced secretion of both pro-inflammatory mediators, and in this way, it shows its anti-inflammatory properties [12, 14, 25]. Additionally, a recent study suggests PEITC and SFN can be useful in treating AIDSrelated heart dysfunction by reducing iNOS expression and the NF- $\mathrm{KB}$ signaling pathway [28]

\section{Benzyl isothiocyanate (BITC)}

One of the hydrolyzed products of glucotropaeolin is BITC which is commonly found in garden cress, and Indian cress. The compound depicted a preventive effect on various cancers such as brain tumors by suppressing NF- $\mathrm{kB}$ [10]. Also, BITC possesses anti-inflammatory effects via downregulation of NF- $\mathrm{KB}$ signaling [13].

\section{Allyl isothiocyanate (AITC)}

Allyl ITC (AITC) is an ITC derived from sinigrin, a type of GS found in horseradish, brussels sprout, cabbage, cauliflower, black mustard, and wasabi. According to reports, sinigrin has different therapeutic activities such as anticancer, anti-inflammatory, antibacterial, antifungal, antioxidant, and wound healing effects [29, 30]. AITC is known to be involved in the regulation of the NF- $\kappa B$-mediated inflammatory cascade in cancer pathology. Also, according to a research, anti-neuroinflammatory effects of AITC occurs through the inhibition of TNF- $\alpha$ and IL- 6 producing by a reduction of NF- $\kappa \mathrm{B}-$ mediated transcription [29]. Other studies have described that AITC reduces cerebral infarction in two ways, one by decreasing the levels of pro-inflammatory molecules including IL-1 $\beta$, IL-6 through the downregulation of NF- $\mathrm{BB}$, and in another way, by upregulating the Nrf2 pathway and subsequently increasing antioxidative mechanisms [13, 31].

\section{6-(Methylsulfinyl) hexyl isothiocyanate (6-MSITC)}

6-MSITC is produced through enzymatic hydrolysis of glucohesperin, which is usually abundant in cruciferous vegetables such as wasabi [25, 32]. Several studies demonstrated that 6-MSITC has anti-inflammatory, antimicrobial, antiplatelet, and anticancer effects. 6-MSITC plays an anti-inflammatory effect in a variety of ways, directly or indirectly regulating the transcription of inflammatory genes, such as COX-2, iNOS, TNF$\alpha$, IL-1 $\beta$, and IL-6 [32]. The modulation of Nrf2 activity within the nucleus is an important way for 6-MSITC to inhibit the production of inflammatory factors [25].

\section{Moringin}

4-( $\alpha$-1-rhamnosyloxy) benzyl ITC, is a type of ITC called moringin, which is produced by myrosinase-catalyzed hydrolysis of glucomoringin (GMG) found in the Moringaceae family. It shows that moringin has a wide range of biological activities such as anti-inflammatory, antioxidant, antimicrobial and antitumor. Besides, this compound clearly can be helpful to counteract the inflammatory cascade that leads to severe multiple sclerosis (MS) [33]. Other cancer studies have shown that moringin not only inhibits oxidative stress by expressing Nrf2, but is more effective than sulforaphane in inhibiting NF-kB activity [34].

\section{Goitrin}

Isatidis Radix (dried root of Isatis indigotica) is an important traditional Chinese medicine for the prevention and treatment of a wide range of viral infectious diseases. For example, Isatidis Radix and its preparations have been used in prevention and treatment against SARS in 2003 and H1N1 influenza outbreaks in 2009. This plant belongs to Brassicaceae family and contains GSs such as epiprogoitrin and progoitrin [18, 35-37]. Recent study showed epiprogoitrin, progoitrin and their enzymatic hydrolysis products derived from Isatidis Radix exhibit dose-dependent inhibitory effect against Influenza A virus (H1N1) without toxicity [37]. Meanwhile another study showed that enzymatic degradation product of progoitrin and goitrin selectively elicit an anti-inflammatory response by reducing TNF- $\alpha$ secretion in human peripheral mononuclear blood cells [18].

\section{COVID-19 and GSs, GSHPs}

Clinical evidences show that sulforaphane, in addition to its inhibitory effect on NF-KB and activating effect on Nrf2, downregulates inducible proinflammatory enzymes such as cyclooxygenase (COX-2) and NO synthase (iNOS) and thus modulates many cancer-related events [38]. Another study 
of 40 healthy overweight subjects has demonstrated the consumption of broccoli sprouts containing glucoraphanin during 10 weeks significantly reduces IL-6 and C-reactive protein levels [39]. A recent case study has shown that oral administration of capsule containing broccoli seeds and glucoraphanin as a (Nrf2)-interacting nutrient from the onset of COVID-19 symptoms was effective in improving many symptoms such as cough, nasal, congestion and fatigue. High doses of this capsule were always effective within minutes, even during the cytokine storm caused by COVID-19. These experimental clinical cases support the hypothesis that glucoraphanin is efficient against COVID19 [16]. Besides, evidences suggest that Isatidis Radix as a traditional Chinese medicine is effective in counteracting the inflammation and immune response induced by COVID-19 by affecting the IL-17 signaling pathway and the TNF signaling pathway [40].

\section{Conclusion}

According to this review, GSs and GSHPs, as valuable nutraceuticals, may be useful in prevention and treatment of COVID-19-related cytokine storms via their anti-inflammatory and antioxidant activities. Definite confirmation of their effectiveness requires further studies.

\section{Declarations}

Ethical approval Not applicable, because this article does not contain any studies with human or animal subjects.

Informed consent Not applicable, because this article does not contain any studies with human or animal subjects.

Conflict of interests The author(s) declared no potential conflicts of interest with respect to the research, authorship, and/or publication of this article.

\section{References}

1. Meidaninikjeh S, Sabouni N, Marzouni HZ, Bengar S, Khalili A, Jafari R. Monocytes and macrophages in COVID-19: Friends and foes. Life Sci. 2021;269:119010.

2. Eftekhari M, Salehi A, Enayati A. Management of COVID-19 by phytotherapy: a pharmacological viewpoint. J Rep Pharma Sci. 2021;10(1):153.

3. Doustimotlagh AH, Eftekhari M. Glucose-6-phosphate dehydrogenase inhibitor for treatment of severe COVID-19: Polydatin. Clin Nutr ESPEN. 2021;43:197-9.

4. Choudhary S, Sharma K, Silakari O. The interplay between inflammatory pathways and COVID-19: a critical review on pathogenesis and therapeutic options. Microb Pathog. 2021;150:104673.
5. Kunnumakkara AB, Rana V, Parama D, Banik K, Girisa S, Sahu $\mathrm{H}$, et al. COVID-19, cytokines, inflammation, and spices: How are they related? Life Sci. 2021;284:119201.

6. Patel S, Saxena B, Mehta P. Recent updates in the clinical trials of therapeutic monoclonal antibodies targeting cytokine storm for the management of COVID-19. Heliyon. 2021;7(2):e06158.

7. Liskova A, Samec M, Koklesova L, Samuel SM, Zhai K, Al-Ishaq RK, et al. Flavonoids against the SARS-CoV-2 induced inflammatory storm. Biomedicine \& Pharmacotherapy. 2021;138:111430.

8. Lin C-Y, Yao C-A. Potential role of Nrf2 activators with dual antiviral and anti-inflammatory properties in the management of viral pneumonia. Infect Drug Resist. 2020;13:1735.

9. Kohandel Z, Farkhondeh T, Aschner M, Samarghandian S. Nrf2 a molecular therapeutic target for Astaxanthin. Biomed Pharmacotherapy. 2021;137:111374.

10. Soundararajan P, Kim JS. Anti-carcinogenic glucosinolates in cruciferous vegetables and their antagonistic effects on prevention of cancers. Molecules. 2018;23(11):2983.

11. Zinovkin R, Grebenchikov O. Transcription factor Nrf2 as a potential therapeutic target for prevention of cytokine storm in COVID-19 patients. Biochem Mosc. 2020;85(7):833-7.

12. Cho HJ, Lee KW, Park JHY. Erucin exerts anti-inflammatory properties in murine macrophages and mouse skin: possible mediation through the inhibition of $\mathrm{NF \kappa B}$ signaling. Int J Mol Sci. 2013;14(10):20564-77.

13. Esteve M. Mechanisms underlying biological effects of cruciferous glucosinolate-derived isothiocyanates/indoles: a focus on metabolic syndrome. Front Nutr. 2020;7:111.

14. Cheung KL, Kong A-N. Molecular targets of dietary phenethyl isothiocyanate and sulforaphane for cancer chemoprevention. AAPS J. 2010;12(1):87-97.

15. Horowitz RI, Freeman PR. Three novel prevention, diagnostic, and treatment options for COVID-19 urgently necessitating controlled randomized trials. Med Hypotheses. 2020;143:109851.

16. Bousquet J, Le Moing V, Blain H, Czarlewski W, Zuberbier T, de la Torre R, et al. Efficacy of broccoli and glucoraphanin in COVID-19: from hypothesis to proof-of-concept with three experimental clinical cases. World Allergy Organ J. 2021;14(1):100498.

17. Bones AM, Rossiter JT. The enzymic and chemically induced decomposition of glucosinolates. Phytochemistry. 2006;67(11):1053-67.

18. Tran HT, Herz C, Ruf P, Stetter R, Lamy E. Human T2R38 bitter taste receptor expression in resting and activated lymphocytes. Front Immunol. 2018;9:2949.

19. Maina S, Misinzo G, Bakari G, Kim H-Y. Human, animal and plant health benefits of glucosinolates and strategies for enhanced bioactivity: a systematic review. Molecules. 2020;25(16):3682.

20. Mahn A, Castillo A. Potential of Sulforaphane as a natural immune system enhancer: a review. Molecules. 2021;26(3):752.

21. Patel V, Dial K, Wu J, Gauthier AG, Wu W, Lin M, et al. Dietary antioxidants significantly attenuate hyperoxia-induced acute inflammatory lung injury by enhancing macrophage function via reducing the accumulation of airway HMGB1. Int J Mol Sci. 2020;21(3):977.

22. Cedrowski J, Dąbrowa K, Przybylski P, Krogul-Sobczak A, Litwinienko G. Antioxidant activity of two edible isothiocyanates: Sulforaphane and erucin is due to their thermal decomposition to sulfenic acids and methylsulfinyl radicals. Food Chem. 2021;353:129213.

23. Giacoppo S, Galuppo M, Iori R, De Nicola GR, Cassata G, Bramanti P, et al. Protective role of (RS)-glucoraphanin bioactivated with myrosinase in an experimental model of multiple sclerosis. CNS Neurosci Ther. 2013;19(8):577-84.

24. Gasparello J, D’Aversa E, Papi C, Gambari L, Grigolo B, Borgatti $M$, et al. Sulforaphane inhibits the expression of interleukin-6 and interleukin-8 induced in bronchial epithelial IB3-1 cells 
by exposure to the SARS-CoV-2 Spike protein. Phytomedicine. 2021;87:153583.

25. Jaafaru MS, Abd Karim NA, Enas ME, Rollin P, Mazzon E, Abdull Razis AF. Protective effect of glucosinolates hydrolytic products in neurodegenerative diseases (NDDs). Nutrients. 2018;10(5):580.

26. Shibata T, Nakashima F, Honda K, Lu Y-J, Kondo T, Ushida Y, et al. Toll-like receptors as a target of food-derived anti-inflammatory compounds. J Biol Chem. 2014;289(47):32757-72.

27. Ernst IM, Palani K, Esatbeyoglu T, Schwarz K, Rimbach G. Synthesis and Nrf2-inducing activity of the isothiocyanates iberverin, iberin and cheirolin. Pharmacol Res. 2013;70(1):155-62.

28. Ho J-N, Yoon H-G, Park C-S, Kim S, Jun W, Choue R, et al. Isothiocyanates ameliorate the symptom of heart dysfunction and mortality in a murine AIDS model by inhibiting apoptosis in the left ventricle. J Med Food. 2012;15(9):781-7.

29. Subedi L, Venkatesan R, Kim SY. Neuroprotective and antiinflammatory activities of allyl isothiocyanate through attenuation of JNK/NF-KB/TNF- $\alpha$ signaling. Int J Mol Sci. 2017;18(7):1423.

30. Mazumder A, Dwivedi A, Du Plessis J. Sinigrin and its therapeutic benefits. Molecules. 2016;21(4):416.

31. Caglayan B, Kilic E, Dalay A, Altunay S, Tuzcu M, Erten F, et al. Allyl isothiocyanate attenuates oxidative stress and inflammation by modulating Nrf2/HO-1 and NF- $\kappa \mathrm{B}$ pathways in traumatic brain injury in mice. Mol Biol Rep. 2019;46(1):241-50.

32. Uto T, Hou D-X, Morinaga O, Shoyama Y. Molecular mechanisms underlying anti-inflammatory actions of 6-(methylsulfinyl) hexyl isothiocyanate derived from wasabi (Wasabia japonica). Adv Pharmacol Sci. 2012;2012:614046.

33. Galuppo M, Giacoppo S, De Nicola GR, Iori R, Navarra M, Lombardo GE, et al. Antiinflammatory activity of glucomoringin isothiocyanate in a mouse model of experimental autoimmune encephalomyelitis. Fitoterapia. 2014;95:160-74.

34. Brunelli D, Tavecchio M, Falcioni C, Frapolli R, Erba E, Iori R, et al. The isothiocyanate produced from glucomoringin inhibits $\mathrm{NF}-\mathrm{kB}$ and reduces myeloma growth in nude mice in vivo. Biochem Pharmacol. 2010;79(8):1141-8.

35. Xie Z, Shi Y, Wang Z, Wang R, Li Y. Biotransformation of glucosinolates epiprogoitrin and progoitrin to $(\mathrm{R})$-and $(\mathrm{S})$-goitrin in Radix isatidis. J Agric Food Chem. 2011;59(23):12467-72.

36. Guo Q, Sun Y, Tang Q, Zhang H, Cheng Z. Isolation, identification, biological estimation, and profiling of glucosinolates in Isatis indigotica roots. J Liq Chromatogr Relat Technol. 2020;43(15-16):645-56.

37. Nie L-x, Wu Y-1, Dai Z, Ma S-c. Antiviral activity of Isatidis Radix derived glucosinolate isomers and their breakdown products against influenza $\mathrm{A}$ in vitro/ovo and mechanism of action. $\mathrm{J}$ Ethnopharmacol. 2020;251:112550.

38. Houghton CA. Sulforaphane: its "coming of age" as a clinically relevant nutraceutical in the prevention and treatment of chronic disease. Oxidative Med Cell Longev. 2019;2019:2716870.

39. López-Chillón MT, Carazo-Díaz C, Prieto-Merino D, Zafrilla P, Moreno DA, Villaño D. Effects of long-term consumption of broccoli sprouts on inflammatory markers in overweight subjects. Clin Nutr. 2019;38(2):745-52.

40. Yu B, Lin F, Ning H, Ling B. Network pharmacology study on the mechanism of the Chinese medicine Radix Isatidis (Banlangen) for COVID-19. Medicine. 2021;100(32).

Publisher's note Springer Nature remains neutral with regard to jurisdictional claims in published maps and institutional affiliations. 